\title{
EXPLORATIONS OF THE LANDSCAPE
}

FRANÇOIS JULLIEN:

LIVING OFF LANDSCAPE OR THE UNTHOUGHT-OF IN REASON

TRANSLATED BY PEDRO RODRIGUEZ

LONDON AND NEW YORK:

ROWMAN \& LITTLEFIELD INTERNATIONAL, 2018. 135 PAGES

ISBN 978-1-78660-338-8

A landscape-or the landscape-in its full potential still seems to be at least partially hidden to the viewer. This might be a surprising claim, as it may first look as one of the most straightforward phenomena: it is "there," we can "see” it, perceive and experience it through various modes and forms, it is "in front of us," ready to be appreciated, analysed, recorded, or even transformed. The fact that there is nevertheless a myriad of undiscovered potentials of the landscape is also curious if we consider how often landscapes are visited, researched, described, painted, or even planned, created and re-created over the centuries. Nevertheless, and despite the several hundreds of years of its representation in various forms of visual and fine arts, descriptions in the literary arts and also of its theoretical analyses dedicated to the subject, it still feels-especially after having read François Jullien's latest book on the topic-that it has much more to offer than what we have thought so far. Part of the lack of understanding comes from the comfort of relying on previous results and on being perhaps less motivated in going beyond that-hence it is not surprising that in the beginning of his book, Jullien often uses the term "all too well known” (e.g. pp. 3, 5, 9), describing the possible difficulties of knowing more: i.e. that exactly the established knowledge itself may pose some obstacles on the further discovering and understanding.

"We have ground to believe (or at least a hint of evidence, as I see it) that a transcultural, transhistorical, philosophically useful concept of landscape is possible, and that we needn't settle for the idea that landscape, like a moment in art, is the product of a culturally and historically limited artialization.” (p. 101). This statement comes perhaps surprisingly towards the end of the book, even if it sounds more like a key initial statement in the 
considerations of the author. At the same time, however, instead of placing it in the beginning of the book, and thus risk making it look like a rhetorical trick of grasping the attention of the reader, it summarises the methodical aims of the volume. The particularities of method become crucial in the entire text, and for those less familiar with Jullien's work, the translator Pedro Rodriguez's careful footnotes definitely help as commentaries explaining not only the special terms Jullien uses in his investigation but also in demonstrating how they make up the structure of his thought.

This "transcultural, transhistorical, philosophically useful concept of landscape" is searched and developed with the analyses of the Western and Chinese landscape tradition in painting, poetry, and theoretical texts. Nevertheless, Jullien's method is not comparison in the sense of translating and describing the particularities of common phenomena, or looking for equivalent or relatively similar concepts in the different cultural traditions and showing their possible interconnection. He is much more driven by an active and creative juxtaposition of the different approaches along a divide (écart) in order that they have a mutual reflection on each other. In other words, as Pedro Rodriguez summarised in his commentary note, by placing them "on either side of an exploratory divide" cultures "reveal each other's biases-or, to use another of Jullien's images, they discover each other's cultural headwaters-and thus bring forth new possibilities." (p. ix). Throughout the discussion, this will then turn into an invitation-or even a critical and crucial adviseto pursue an examination in two directions: to go backwards and (re)discover the origins of the phenomena, but also to go forward and to bring forth what is found, i.e. setting this knowledge and understanding gained from the revelatory reflections of the different cultural phenomena to see what can be developed from them, especially from what we may have missed or lost along the way: "Through this mutual face-off we can venture back into their [the conceptions-Z. S.] unthought-of.” (p. 21). Therefore, this double direction of the investigation characterises Jullien's method of cleaning and recovering long-forgotten potentials that lay hidden in the phenomena, and that, again, will help not only in the reconstruction of the meaning and changes of meanings of the concepts, but also in the more precise understanding of the current state of the phenomena too.

If following Jullien's considerations, then I need to admit that even my first sentence in this very review ("A landscape ... hidden 
to the viewer.") contains an issue or bias, since his departure point is exactly the critical investigation of the Western tradition of "viewing" the landscape, i.e. considering it as an object of vision and what can be primarily perceived through the faculty of sight. When focusing on retracing the origins of the emphasis of the visual aspect of landscape-perception typical in Western thought, he identifies it as a key problem that automatically leads to the subject's dominating the landscape, unlike in Chinese thought, where a more comprehensive and more complete experience of landscape is set forth. Revisiting not only the origins of the term in its etymology but also presenting the earliest descriptions and theories of landscape-that in China dates back several centuries earlier than the first European analyses-he unfolds the most characteristic features of the Chinese approach to landscape through profound analyses of the term itself. Two characters "mountain(s)-water(s)" is how "landscape" is expressed in Chinese, and this allows Jullien to develop a convincing interpretation of how in Chinese culture the landscape is not an object of vision, a cut-off of the horizon, but rather a confrontation and correlation of various oppositions (dry and wet, high and low, vertical and horizontal, immobile and flowing etc.) that nevertheless respond to each other. In this way, by breaking with the Western tradition of the domination of and by the dominating power of sight-that naturally also detaches the observer from the landscape-the subject may remain amidst and in between, i.e. instead of being an external viewer, the subject will be immersed in the landscape through the aforementioned interactions of the opposite factors.

Naturally, this will have several philosophical consequences related to the interpretation of the nature of the experience too. Regarding this above immersion, Jullien points out that it is not necessarily an aesthetic category: "Is this an 'aesthetic' pleasure, as the West has categorized it? No. It is, rather, a gratification of living, through the activation it provokes." (p. 39, italics and quotation marks in the original-Z. S.) This will then result not only in the possibility of a novel encountering of the landscape but in the subject's (re)establishment in the world.

Another inspiring part of the volume is when the above considerations are applied to the understanding of other phenomena connected to the perception of natural forms, namely the garden and the kiosk. While the tradition of the French garden-due to the Western dominance of sight and visionfocuses on how the cured and planned section of Nature should look like, thus arbitrarily imposing geometry on it, the Chinese 
garden is more "recreational," in both senses of the word, restoring one's energy by recreating the tensions experienced in an actual landscape. In a similar way, kiosks (ting in Chinese), small constructions placed in the landscape without the function of for example dwelling or commercial hospitality services, also serve to promote a submersion in the landscape, instead of simply providing an observation point.

Jullien's main merit with this book, therefore, lies not (only) in the explanation of the differences of the cultural tradition and their landscape-interpretation, and not even merely in the fact of presenting his method of investigating the concepts through the divide, but that through his observation of the tradition he manages to propose novel ways of being engaged with the landscape that have multiple further consequences not only in interpreting past art products and cultural phenomena but also in offering new answers to contemporary environmental and ecological issues.

Zoltán Somhegyi 